\title{
Efeito da concentração da solução nutritiva no crescimento da alface em cultivo hidropônico-sistema NFT ${ }^{1}$
}

\author{
Nilton Nélio Cometti'; Gean Carlos S Matias ${ }^{4}$; Everaldo Zonta ${ }^{2}$, Wellington Mary ${ }^{3}$, Manlio S Fernandes ${ }^{2}$ \\ ${ }^{1}$ Escola Agrotécnica Federal de Colatina, BR 259, km 70, 29709-910 Colatina-ES; ${ }^{2}$ UFRRJ-Instituto de Agronomia, Depto. Solos, BR \\ 465, km 07, 23890-000 Seropédica-RJ. ${ }^{3}$ UFRRJ-Instituto de Tecnologia, Depto. Arquitetura e Urbanismo. ${ }^{4}$ Doutorando USP-ESALQ, C. \\ Postal 09, 13418-900 Piracicaba-SP; nilton@eafcol.gov.br; www.niltoncometti.com.br
}

\section{RESUMO}

Foi avaliado o crescimento da alface (Lactuca sativa L.) cv 'Vera' em um sistema hidropônico NFT com diferentes concentrações da solução nutritiva. $\mathrm{O}$ experimento foi realizado em casa de vegetação de abril a maio de 2000, na UFRRJ, Seropédica (RJ). Os tratamentos constaram de quatro soluções nutritivas, diferentes quanto à concentração de macronutrientes: 100; 50; 25 e 12,5\% da concentração original proposta por Furlani (1997). A produção de massa seca (parte comercial) em solução nutritiva a $50 \%$ da concentração original, e condutividade elétrica em torno de $0,98 \mathrm{dS} \mathrm{m}^{-1}$, foi semelhante à solução a $100 \%$ da força iônica. As produções obtidas com 100 e $50 \%$ da força iônica estiveram dentro da faixa de $90 \%$ da produção máxima, calculada em $75 \pm 1 \%$ da força iônica da solução nutritiva, simulada por equação de regressão. Entretanto, o uso de concentrações menores resultou em redução no crescimento de $50 \%$ com a solução a $25 \%$ e $80 \%$ com a solução a $12,5 \%$ da força iônica, nas condições de luz e temperatura em que o experimento foi conduzido. A redução da concentração da solução nutritiva permite uma economia de pelo menos $50 \%$ no custo da solução nutritiva básica, reduzindo-se a solução inicial para $1,00 \mathrm{dS} \mathrm{m}^{-1}$, sem comprometer a produtividade.

Palavras-chave: Lactuca sativa L., condutividade elétrica, taxa de crescimento, força iônica.

\section{ABSTRACT}

Effects of the concentration of nutrient solution on lettuce growth in hydroponics-NFT system

The growth of lettuce (Lactuca sativa L.) cv 'Vera' was evaluated in a NFT hydroponic system with different nutrient solution concentrations. An experiment was carried out in a greenhouse from April to May, 2000 in Rio de Janeiro State, Brazil. Four treatments were used differing in macro nutrient concentration: 100; 50; 25 and $12.5 \%$ of the original concentration of the solution proposed by Furlani (1997). Using $50 \%$ of the original concentration with electric conductivity around $0,98 \mathrm{dS} \mathrm{m}$, plants produced the same shoot dry mass as using $100 \%$ of the ionic strength. In this trial, the phytomass yield using 100 and $50 \%$ of the ionic strength was within the range of $90 \%$ of the maximum production, calculated at $75 \pm 1 \%$ of the ionic strength to the nutrient solution, simulated by a regression equation. However, the use of smaller concentrations resulted in reduction of the growth of $50 \%$ with the solution at $25 \%$ and $80 \%$ with the solution at $12.5 \%$ of the ionic strength, under the light and temperature conditions which the experiment was carried out. Decreasing the nutrient solution concentration one can reduce up to $50 \%$ of the basic nutrient solution costs, if nutrient solution concentration is reduced and electric conductivity kept close to $1.00 \mathrm{dS} \mathrm{m} \mathrm{m}^{-1}$.

Keywords: Lactuca sativa L., electric conductivity, growth rate, ionic strength.

\section{(Recebido para publicação em 16 de abril de 2007; aceito em 23 de abril de 2008)}

$\mathrm{O}$ cultivo hidropônico da alface temse difundido rapidamente em todo o país. Na região Sudeste, em especial, tem alcançado seu máximo crescimento, o que pode ser atribuído a uma série de fatores, destacando-se o melhor preço final do produto, maior demanda por produtos de qualidade superior e maior difusão de tecnologia. O sistema hidropônico chamado NFT (técnica de nutrientes em filme) tem alcançado destaque na produção desta hortaliça, sendo o preferido dentre os vários sistemas disponíveis pelas vantagens de praticidade e eficácia na produção (Cometti, 2003).

Muitas fórmulas de soluções nutritivas têm sido usadas e avaliadas quan- to à produtividade. Trabalhos de Santos (1998) mostram que não há grande diferença na produtividade entre as soluções nutritivas comumente utilizadas.

Em geral, as soluções nutritivas em voga têm como ancestral comum a solução proposta por Hoagland \& Arnon em 1938 (Hoagland \& Arnon, 1950), cujos níveis de macro e micronutrientes muito se assemelham aos atualmente preconizados. A elevada concentração de sais observada na solução de Hoagland \& Arnon (1950) se perpetuou e pode ser observada na maioria das soluções em uso atualmente, alcançando níveis de condutividades elétricas (CE) maiores do que 2,0 dS m-1. Costa et al. (2001), cultivando alface americana de cabeça crespa, cultivar Ryder, em hidroponia, observaram as maiores produtividades em soluções com 2,46 \pm $0,24 \mathrm{dS} \mathrm{cm}^{-1}$, nas condições de Botucatu, SP. Entretanto, o uso de concentrações salinas elevadas nas soluções nutritivas, sob condições ambientais de alta temperatura, alta umidade e elevada luminosidade, como as que ocorrem nos estados do Rio de Janeiro e Espírito Santo, têm provocado situações inusitadas que não ocorrem normalmente em clima temperado. Dentre elas, distúrbios fisiológicos das plantas podem ser considerados como os mais preocupantes, expressando-se na forma de murcha

${ }^{1}$ Trabalho parcial da Tese de Doutorado do primeiro autor. 
excessiva nas horas mais quentes do dia, queima das bordas das folhas "tipburn" e perda na produtividade (Huett, 1994). É crescente a preocupação em reduzir a concentração das soluções nutritivas, por vários motivos: redução da concentração de nitrato nos tecidos vegetais; redução do potencial de eutroficação das soluções remanescentes dos cultivos hidropônicos (Siddiqi et al., 1998); e redução dos custos de produção por meio do aumento da eficiência do uso do nutriente. Trabalhos de Siddiqi et al. (1998) e Chen et al. (1997) mostram ser possível reduzir a concentração da solução nutritiva a níveis tão baixos quanto $10 \%$ da força iônica original das soluções comumente usadas em cultivos hidropônicos em sistemas recirculantes de alface e tomate, sem que se incorra em riscos de perda da produtividade.

Diante do exposto, o objetivo deste trabalho foi avaliar o crescimento e a produtividade da alface cultivada em sistema hidropônico-NFT, com diferentes concentrações de macronutrientes na solução nutritiva.

\section{MATERIAL E MÉTODOS}

Alface (Lactuca sativa L.), cultivar Vera, foi cultivada em um sistema hidropônico do tipo NFT, de abril a maio, na UFRRJ, Seropédica (RJ) (coordenadas geográficas $22^{\circ} 45^{\prime} 33^{\prime \prime} \mathrm{S}$ e $43^{\circ} 41^{\text {' }}$ $51^{\prime \prime)}$. Os tratamentos constaram de quatro soluções nutritivas diferentes quanto a concentração de macronutrientes, variando de 100 a 12,5\% da concentração original da solução proposta por Furlani (1997), conforme a Tabela 1. Os micronutrientes foram mantidos na mesma concentração em todos os tratamentos: $\mathrm{B}=0,3, \quad \mathrm{Cu}=0,02, \quad \mathrm{Fe}=2, \quad \mathrm{Mn}=0,4$, $\mathrm{Mo}=0,06$ e $\mathrm{Zn}=0,06 \mathrm{mg} \mathrm{L}^{-1}$. As soluções de macronutrientes foram preparadas a partir de sais comerciais indicados para fertirrigação (nitrato de potássio, nitrato de cálcio, fosfato mono-amônio e sulfato de magnésio). Para os micronutrientes foram utilizados sais puros. O ferro foi utilizado na forma de quelato EDTA $(13 \% \mathrm{Fe})$. Todas as soluções foram preparadas com água destilada.

O sistema hidropônico completo constou de quatro subsistemas indepen- dentes, com quatro canais de cultivo, contendo 22 plantas em cada canal, considerado como uma repetição. Cada solução (tratamento) foi distribuída em um canal por bancada, de forma aleatória, para evitar o efeito de localização dentro da casa de vegetação. Cada subsistema foi composto de um reservatório de solução nutritiva em polietileno com volume de $100 \mathrm{~L}$, uma motobomba de $32 \mathrm{~W}$, tubulação de recalque de solução em PVC com diâmetro de $25 \mathrm{~mm}$, canal de cultivo em polipropileno do tipo "Hidrogood®" com diâmetro de 75 mm, tubulação de retorno da solução em PVC com diâmetro de $50 \mathrm{~mm}$ e um temporizador/ contator para acionamento elétrico das motobombas.

A semeadura foi realizada em espuma fenólica mantida úmida com água destilada por três dias, a partir dos quais as células de espuma fenólica com as mudas foram destacadas e colocadas nos canais de cultivo. A partir dessa data receberam a solução proposta por Furlani (1997) diluída a 25\% da concentração original até os 17 dias da semeadura. Aos 17 dias após a semeadura (DAS) as plantas receberam as soluções nas concentrações definitivas nos tratamentos. A vazão da solução nutritiva nos canais de cultivo foi de $90 \mathrm{~L} \mathrm{~h}^{-1}$. A freqüência de funcionamento foi de 10 minutos com intervalos de 10 minutos durante todo o dia, até o final do experimento.

Diariamente, os níveis das soluções nos reservatórios foram reajustados com água destilada e a condutividade elétrica com soluções estoques para manter a concentração inicial do tratamento, não sendo permitidas oscilações maiores do que $20 \%$ da concentração inicial da solução. As trocas das soluções nutritivas foram feitas semanalmente para evitar grandes desequilíbrios entre os nutrientes. Utilizou-se $\mathrm{KOH}$ a $0,05 \mathrm{~mol} \mathrm{~L}^{-1} \mathrm{e}$ $\mathrm{H}_{2} \mathrm{SO}_{4}$ a $0,025 \mathrm{~mol} \mathrm{~L}^{-1}$ para corrigir o $\mathrm{pH}$ para 5,5. A temperatura e o fluxo de fótons fotossintéticos (FFF) foram monitorados três vezes ao dia. A temperatura média durante o dia no período foi de $30 \pm 4^{\circ} \mathrm{C}$ e o FFF médio foi de $600 \pm 200 \mu \mathrm{mol} \mathrm{m}{ }^{-2} \mathrm{~s}^{-1}$.

As coletas de plantas foram realizadas em intervalos de sete dias, inician- do-se aos 17 DAS e finalizando aos 52 DAS. O delineamento experimental utilizado foi inteiramente casualizado e cada parcela experimental foi composta de quatro plantas coletadas em cada canal de cultivo, com exceção da primeira, aos 17 DAS, quando foram coletadas apenas duas plantas para formar uma amostra composta, pois todos os sistemas possuíam a mesma solução nutritiva até então. A parte aérea das plantas foi separada em folhas e caules, e juntamente com as raízes foram levados à estufa para secagem a $80^{\circ} \mathrm{C}$ até massa constante.

As Taxa de Crescimento Absoluto (TCA) e Taxa de Crescimento Relativo (TCR) foram calculadas pelas equações (Hunt, 1990):

$$
\begin{gathered}
T C A=\frac{m_{2}-m_{1}}{t_{2}-t_{1}}\left(\mathbf{g ~ d i a}{ }^{-1}\right) \\
T C R=\frac{\left(\log _{e} m_{2}-\log _{e} m_{1}\right)}{t_{2}-t_{1}} \quad\left(\mathbf{g ~ g}^{-1} \mathbf{d i a}^{-1}\right)
\end{gathered}
$$

Onde, $m=$ massa seca (g) e $t=$ tempo em dias.

Os dados foram submetidos à análise de variância em delineamento inteiramente casualizado. Para a análise estatística e análise de regressão utilizouse o programa SigmaStat ${ }^{\circledR}$ (SPSS Science, Chicago, IL).

\section{RESULTADOS E DISCUSSÃO}

As alfaces cultivadas com 100 e 50\% da concentração da solução nutritiva apresentaram os maiores valores de taxa de crescimento absoluto (TCA), chegando a $1,1 \mathrm{~g} \mathrm{dia}^{-1}$ no período compreendido entre 45 e 52 DAS (Figura 1). Já os tratamentos com 25 e $12,5 \%$ da concentração da solução apresentaram menores valores de TCA, da ordem $0,74 \mathrm{e}$ 0,29 $\mathrm{g} \mathrm{dia}^{-1}$ no período de 45 a 52 DAS, respectivamente. Em cultivos convencionais, Garcia et al. (1982) observaram taxas de crescimento de alface da cultivar Brasil 48 em torno de 0,48 $\mathrm{g} \mathrm{dia}^{-1}$ entre os 62 e 72 dias após o transplante. Valores semelhantes foram encontrados por Fernandes et al. (1971). Segundo Garcia et al. (1988), também em cultivo convencional, a cultivar Clause's Aurélia apresentou máxima TCA no período entre os 51 e 62 dias após o transplante, 0,40 $\mathrm{g} \mathrm{dia}^{-1}$, apresentando 




Figura 1. Taxa de crescimento absoluto (TCA) e taxa de crescimento relativo (TCR) da alface cultivada em sistema hidropônico-NFT nas concentrações de 100, 50, 25 e 12,5\% da solução proposta por Furlani (1997). Cada ponto representa a média de quatro repetições. As barras de erro indicam desvio padrão (absolute growth rate (TCA) and relative growth rate (TCR) of lettuce grown in a hydroponic system-NFT in the concentrations of 100, 50, 25 , and $12, ., 5 \%$ of the solution proposed by Furlani (1997). Each point represents the average of four replicates. The error bars indicate standard deviation). Seropédica, UFRRJ, 2000.

ligeiro declínio após esse período. Faquin et al. (1996) cultivando alface Verônica, do tipo crespa, em sistema hidropônico NFT, encontrou um máximo de TCA no período compreendido entre 15 e 20 dias após o transplante, em torno de $1,4 \mathrm{~g} \mathrm{dia}^{-1}$ por planta, mas a maioria dos períodos entre as colheitas apresentou TCA em torno de $0,5 \mathrm{~g} \mathrm{dia}^{-1}$. Como o experimento contou o tempo a partir do transplante de plântulas com cinco folhas, pode-se inferir que as plantas possuíam em torno de 20 dias ao transplante, e com isso, a colheita no ponto comercial foi realizada aos 50 DAS, onde as plantas alcançaram TCA em torno de $0,52 \mathrm{~g} \mathrm{dia}^{-1}$. Para comparações desses resultados com os que foram obtidos neste experimento, deve ser levada em consideração a variação na taxa de crescimento em função, principalmente, das variáveis de temperatura e de luminosidade, que também se alteram ao longo do ano. Entretanto, num aspecto geral, observa-se que no presente trabalho a alface cultivada em hidroponia apresentou precocidade ao alcançar uma TCA de $0,9 \mathrm{~g} \mathrm{dia}^{-1}$, e pa- derivada do crescimento em determinado período. A TCR mostra uma vantagem na interpretação de mudanças no crescimento por não sofrer grandes variações com diferentes períodos analisados (Hunt, 1990). Conseqüentemente, não se observaram diferenças entre as TCR entre nenhum dos tratamentos, tanto para os períodos compreendidos entre 38 a 45 DAS quanto 45 a 52 DAS (Figura 1), visto que a TCR está muito mais relacionada às características genéticas intrínsecas à planta do que às modificações ambientais. Não houve diferença estatística entre as coletas a partir de 31 DAS, com valores em torno de $0,1 \mathrm{~g} \mathrm{~g}^{-1} \mathrm{dia}^{-1}$, mas nota-se que há uma tendência de declínio na TCR dos tratamentos 100,50 e $25 \%$ no período de 45 a 52 DAS, o que indica a aproximação da senescência, momento em que a assimilação líquida passa a ser negativa. Garcia et al. (1982) também encontraram valores de TCR em torno de 0,1 $\mathrm{g} \mathrm{g}^{-1} \mathrm{dia}^{-1}$ aos 41 dias da cultura de alface cultivada em solo.

As maiores produções de massa seca de folha e de caule foram obtidas com os tratamentos 100 e $50 \%$, especialmente nas duas últimas coletas (Figura 2). Aos 45 DAS, esses tratamentos alcançaram em torno de $11 \mathrm{~g}$ de folhas (massa seca) por planta. Esses valores de massa seca em sistema hidropônico são elevados, quando comparados à produção de alface em solo. Garcia et al. (1988), trabalhando com alfaces das cultivares Brasil 48 e Clause's Aurélia cultivadas em solo, obtiveram 12,5 g de massa seca por planta apenas aos 72 dias da cultura. Faquin et al. (1996) obtiveram plantas com 14,8 $\mathrm{g}$ de folhas (em base de massa seca) por planta aos 30 dias após o transplante, no ponto de colheita. Entre os tratamentos 100 e 50\%, no entanto, não houve diferença estatística, indicando que é perfeitamente viável a utilização da solução nutritiva à metade de sua força iônica sem prejuízo da produção final. Em contrapartida, os dados da quarta e da quinta coleta mostram que a diferença na produção de massa seca foliar foi significativa entre os tratamentos 50 e $25 \%$. Apesar da colheita aos 52 DAS (quinta coleta) não ser comum em sistemas de produção hidropônica comercial devido ao 
Tabela 1. Soluções nutritivas dos tratamentos utilizados no experimento. (nutrient solution composition of the treatments used in the trial). Seropédica, UFRRJ, 2000.

\begin{tabular}{|c|c|c|c|c|c|c|c|c|c|c|}
\hline \multirow{2}{*}{ Trat $^{1}$} & $\mathrm{~N}-\mathrm{NO}_{3}-$ & $\mathrm{N}-\mathrm{NH}_{4}+$ & $\mathbf{P}$ & $\mathbf{K}$ & $\mathrm{Ca}$ & $\mathbf{M g}$ & $\mathbf{s}$ & \multirow{2}{*}{$\begin{array}{c}C E^{2} \\
\left(\mathrm{dS} \mathrm{m}^{-1}\right)\end{array}$} & {$[\text { Íons }]^{3}$} & Força lônica ${ }^{4}$ \\
\hline & & & & $\mathrm{mg} \mathrm{L}^{-1}$ & & & & & \multicolumn{2}{|c|}{$\mathrm{mmol} \mathrm{L}^{-1}$} \\
\hline $100 \%$ & 174,0 & 24,0 & 39,0 & 183,0 & 142,0 & 38,0 & 52,0 & 1,84 & 26,92 & 21,94 \\
\hline $50 \%$ & 87,0 & 12,0 & 19,5 & 91,5 & 71,0 & 19,0 & 26,0 & 0,98 & 13,52 & 11,22 \\
\hline $25 \%$ & 43,5 & 6,0 & 9,8 & 45,8 & 35,5 & 9,5 & 13,0 & 0,54 & 6,81 & 5,73 \\
\hline $12,5 \%$ & 21,8 & 3,0 & 4,9 & 22,9 & 17,8 & 4,8 & 6,5 & 0,29 & 3,46 & 2,92 \\
\hline
\end{tabular}

${ }^{1}$ Percentagem da concentração de macronutrientes da solução proposta por Furlani (1997). (percentage of the macronutrients concentration of the solution proposed by Furlani (1997); '2Condutividade elétrica medida com condutivímetro portátil da marca 'Hanna®' (electric conductivity measured by portable conductivity meter Hanna $\left.{ }^{\circledR}\right) ;{ }^{3}$ Concentração total de ions; ${ }^{4}$ Calculada com o auxílio do programa GeochemPC (Sposito \& Mattigod, 1980) (calculatec using Geochem-PC (Sposito \& Mattigod, 1980)

porte das plantas, o presente experimento foi conduzido até esse estádio para fins de conhecimento do efeito da concentração ao longo de toda a ontogenia da planta. $\mathrm{O}$ fato das plantas cultivadas com $50 \%$ da solução nutritiva proposta por Furlani (1997) produzirem 65\% mais massa seca foliar do que o tratamento com $25 \%$, e de produzirem o mesmo que o tratamento $100 \%$, indica que $50 \%$ da concentração da solução encontra-se próximo ao limite e que uma diluição na solução poderia implicar na perda de produtividade pela cultura. No sistema radicular, entretanto, o efeito da concentração da solução nutritiva mostra-se linear, com diferenças entre os quatro tratamentos. Isso indica que o crescimento do sistema radicular é reduzido com a diluição da solução nutritiva, porém sem afetar a produção de fitomassa, até a CE de $1,0 \mathrm{mS} \mathrm{m}^{-1}$, aparentando uma economia de carbono utilizado para o crescimento do sistema radicular. Do ponto de vista comercial, esse efeito é vantajoso para a produção de folhosas como a alface.

A Figura 3 mostra o efeito da concentração da solução na duas últimas datas de coletas, consideradas de interesse comercial (Cometti, 2003). Em ambas, as produções máximas obtidas pelas equações de regressão foram alcançadas na concentração de $75 \pm 1 \%$. Entretanto, os tratamentos 100 e $50 \%$ produziram massas muito próximas a 90\% do máximo (linhas de referências na Figura 3), aceitável para a produtividade de uma cultura. Aos 45 DAS, as reduções foram de 14 e $11,5 \%$, e aos 52 DAS foram de 12,6 e 12,2\% para as concentrações de 50 e $100 \%$, respectivamente, em relação ao máximo calcu-

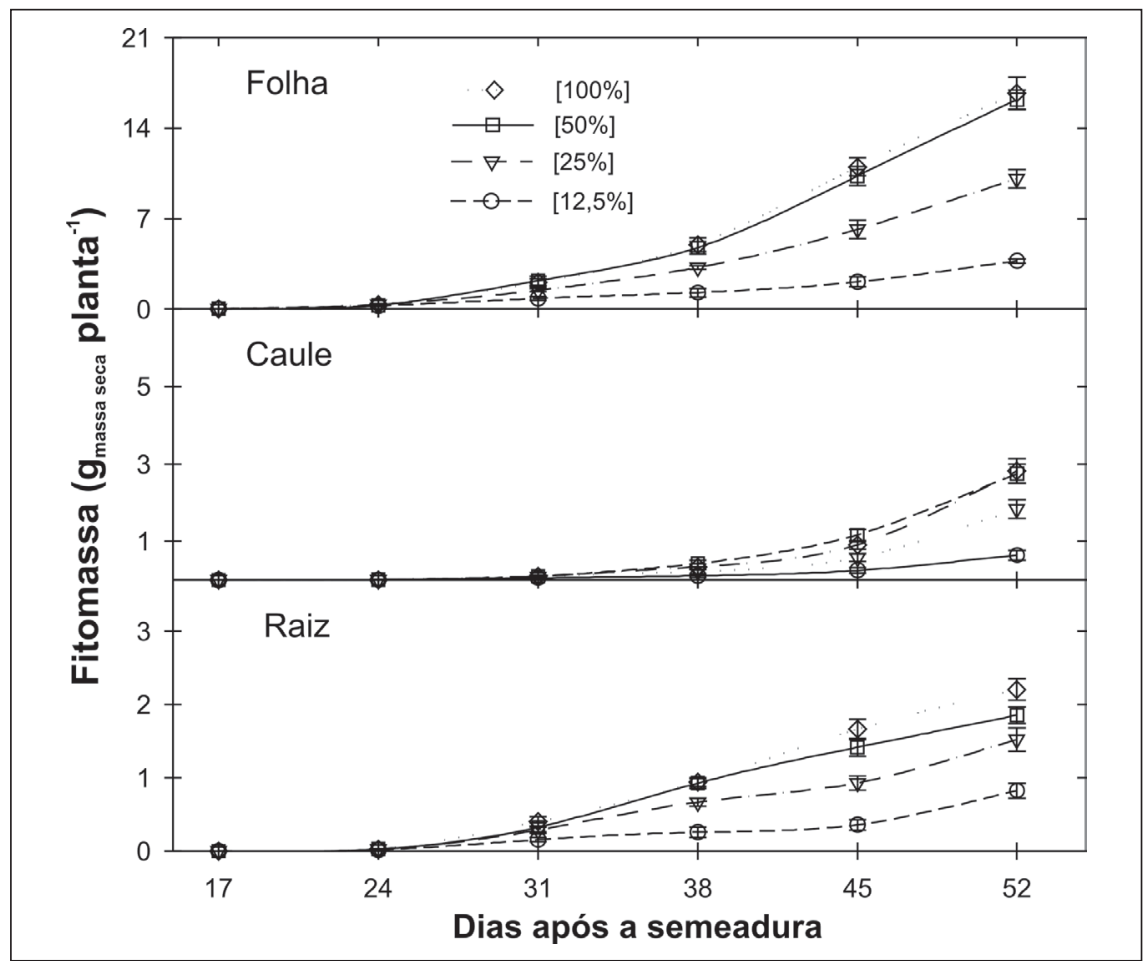

Figura 2. Produção de fitomassa pela alface cultivada em sistema hidropônico-NFT nas concentrações de 100, 50, 25 e 12,5\% da solução proposta por Furlani (1997). Cada ponto representa a média de quatro repetições. As barras de erro indicam desvio padrão. (phytomass production by lettuce grown in a hydroponic system-NFT in the concentrations of 100, 50, 25 , and $12.5 \%$ of the solution proposed by Furlani (1997). Each point represents the average of four replicates. The error bars indicate standard deviation). Seropédica, UFRRJ, 2000.

lado a partir das curvas de ajuste. Esses resultados trazem uma nova compreensão sobre a faixa apropriada de trabalho da concentração da solução, que pode variar, no caso da alface, de 100 a 50\% da concentração inicialmente proposta, sem prejuízo da produtividade da cultura. Entretanto, maiores diluições podem causar rápido decréscimo na produtividade (Figuras 2 e 3 ), que passa a ser reduzida de forma proporcional e linear a concentração da solução nutritiva. A manutenção de uma condutividade elétrica constante equivalente a $75 \%$ da concentração da solução é difícil na prática das hidroponias comerciais, pois há diluição da solução ao longo do dia, quando da entrada de água por válvula bóia, ou concentração quando a água é adicionada apenas junto com a adição dos nutrientes. Entretanto, aparentemente, manter as diluições até $50 \%$ da concentração inicial da solução proposta por Furlani (1997), com a condutividade em torno de $1,0 \mathrm{dS} \mathrm{m}^{-1}$, é factível, mantendo a produtividade da cultura de alface 


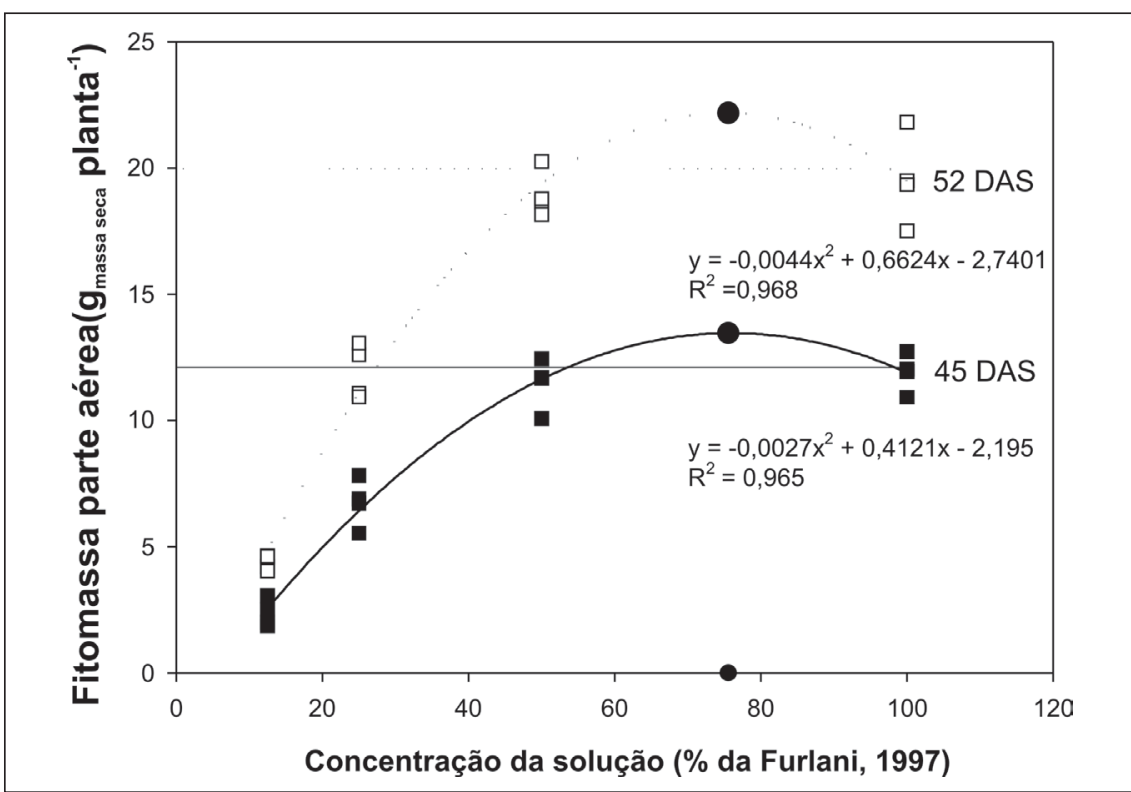

Figura 3. Produção de fitomassa pela alface cultivada em sistema hidropônico-NFT em função da concentração da solução nutritiva em coletas aos 45 e 52 dias após a semeadura (DAS). Cada ponto representa a média de quatro plantas. Os círculos cheios nas curvas indicam o ponto de máximo calculado através das equações de regressão. (phytomass production by lettuce grown in a hydroponic system-NFT depending on the concentration of the nutrient solution harvest at 45 and 52 days after seeding (DAS). Each point represents the average of four replicates. The error bars indicate standard deviation). Seropédica, UFRRJ, 2000.

hidropônica. Oscilações, da ordem de 0,5 $\mathrm{dS} \mathrm{m}^{-1}$ quando a solução apresenta CE acima de $1,0 \mathrm{dS} \mathrm{m}^{-1}$, não promovem alteração significativa na produtividade.

Têm surgido propostas de baixar a concentração das soluções através do abaixamento da CE para a faixa 1,0 a $1,5 \mathrm{dS} \mathrm{m}^{-1}$ para regiões mais quentes como Norte e Nordeste (Furlani et al., 1999), porém sem dados consistentes para essas regiões, que vêm a ser supridos pelo presente trabalho. Claussen (2002), trabalhando com tomate, observou que variando de 1 a 5 vezes a concentração da solução nutritiva original, sob nutrição totalmente nítrica ou utilizando até $25 \%$ do nitrogênio na forma amoniacal, não houve alteração tanto no crescimento vegetativo quanto no reprodutivo. Do mesmo modo, Genúncio et al. (2006), observaram que diluições da solução de Hoagland \& Arnon (1950) a 50\%, baixando de 2,88 $\mathrm{dS} \mathrm{m}{ }^{-1}$ para $1,44 \mathrm{dS} \mathrm{m}^{-1}$, não influenciaram a produtividade e o acúmulo de massa dos tomateiros.

A redução da concentração da solução nutritiva a $50 \%$, nas condições do presente experimento, pode representar uma economia de $50 \%$ do custo da so- lução nutritiva básica. Comercialmente, encontram-se kits de nutrientes disponíveis prontos para uso, normalmente para $1000 \mathrm{~L}$ de solução (LUZ et al., 2006). Seu custo atual é de $\mathrm{R} \$ 11,00$, obtido por consulta à empresa comercial Gioplanta ${ }^{\circledR}$ em 04/12/07. Numa produção hidropônica de 1 há, se considerada a produção mensal de 50.000 plantas e a utilização de $100000 \mathrm{~L}$ de solução nutritiva básica inicial (2 L plan$\left.\mathrm{ta}^{-1}\right)$, pode-se alcançar uma economia anual de até R \$ 6.000,00 (US\$ 2.790,00 ao câmbio de 2,15:1) em nutrientes. Essa economia seria obtida apenas com a redução da condutividade elétrica da solução inicial de 2,0 para $1,0 \mathrm{dS} \mathrm{m}^{-1}$, sem comprometer a produtividade. Essa inferência vale apenas para regiões com clima semelhante ao deste experimento, com alta temperatura e alto fluxo de fótons fotossintéticos.

A utilização da solução nutritiva proposta de Furlani (1997) a 50\% da concentração original, com condutividade elétrica em torno de $0,98 \mathrm{dS} \mathrm{m}^{-1}$, resultou na produção da mesma massa de parte aérea (parte comercial) do que a solução a $100 \%$ da força iônica, com condutividade elétrica em torno de 1,84
$\mathrm{dS} \mathrm{m}^{-1}$. Com isso, racionaliza-se o uso de fertilizantes e diminuem-se os custos de produção da alface em sistema hidropônico NFT. Nas condições de luz e temperatura em que foi conduzido o experimento, maiores diluições da solução poderiam levar à redução no crescimento. As taxas de crescimento absoluto das plantas submetidas aos tratamentos com 100 e $50 \%$ da concentração da solução foram semelhantes e crescentes ao longo do experimento, alcançando $1,1 \mathrm{~g} \mathrm{dia}^{-1}$. Entretanto, a eficiência de produtividade, ou taxa de crescimento relativo, entrou em declínio após o período de 45 DAS.

\section{AGRADECIMENTOS}

Os autores agradecem ao $\mathrm{CNPq}$ pela bolsa de produtividade e de iniciação científica.

\section{REFERÊNCIAS}

ANDRIOLO JL; GODOI RS; COGO CM; BORTOLOTTO OC; LUZ GL; MADALOZ CJ. 2006. Growth and development of lettuce plants at high $\mathrm{NH}_{4}^{+}: \mathrm{NO}_{3}{ }_{3}^{-}$ratios in the nutrient solution. Horticultura brasileira 24: 352-355.

CARRASCO G; IZQUIERDO JA. 1996. A Média empresa hidropônica: a técnica da solução nutritiva recirculante ("NFT") Talca, Chile: Universidade de Talca/FAO. $91 \mathrm{p}$.

CASTELlANE PD; ARAÚJO JAC. 1995. Cultivo sem solo-hidroponia. 4. ed. Jaboticabal: FUNEP/UNESP. 43 p.

CHEN XG; GASTALDI C; SIDDIQI MY; GLASS ADM. 1997. Growth of a lettuce crop at low ambient nutrient concentrations: a strategy designed to limit the potential for eutrophication. Journal of Plant Nutrition 20: 1403-17.

CLAUSSEN W. 2002. Growth, water use efficiency, and proline content of hydroponically grown tomato plants as affected by nitrogen source and nutrient concentration. Plant and Soil 00: 1-11.

COMETTI NN. 2003. Nutrição mineral da alface (Lactuca sativa L.) em cultura hidropônica sistema NFT. Seropédica: UFRRJ. 128p (Tese doutorado).

COMETTI NN; FURLANI PR; RUIZ HA; FERNANDES FILHO EI. 2006. Soluções Nutritivas: formulação e aplicações. In: MANLIO SF. (ed.). Nutrição Mineral de Plantas. Viçosa, MG: Sociedade Brasileira de Ciência do Solo. p. 89-114.

COSTA PC; DIDONE EB; SESSO TM; CAÑIZARES KAL; GOTO R. 2001. Condutividade elétrica da solução nutritiva e produção de alface em hidroponia. Scientia Agricola 58: 595-597.

FAQUIN V; FURTINI NETO AE; VILELA LAA. 1996. Produção de alface em hidroponia. Lavras: UFLA. 50 p. 
FERNANDES PD; OLIVEIRA GD; HAAG HP. 1971. Nutrição mineral de hortaliças. XIV. Absorção de macronutrientes pela cultura da alface. $O$ solo $63:$ 7-10.

FURLANI PR. 1997. Instruções para o cultivo de hortaliças de folhas pela técnica de hidroponia-NFT. Campinas: Instituto Agronômico. 30 p. (Boletim técnico, 168).

FURLANI PR, SILVEIRA LCP; BOLONHEZI D; FAQUIN V. 1999. Cultivo hidropônico de plantas. Campinas: Instituto Agronômico, 52 p. (Boletim técnico, 180).

GARCIA LLC; HAAG HP; MINAMI K; DECHEN AR. 1982. Nutrição mineral de hortaliças. XLIX. Concentração e acúmulo de macronutrientes em alface (Lactuca sativa $\mathrm{L}$.) cv. Brasil 48 e Clause's Aurélia. Anais da Escola Superior de Agricultura "Luiz de Queiroz" 39: 455-484.

GARCIA LLC; HAAG HP; MINAMI K; DECHEN AR. 1988. Nutrição mineral de hortaliças: concentração e acúmulo de macronutrientes em alface (Lactuca sativa $\mathrm{L}$.) cv. Brasil 48 e Clause's Aurélia. In: HAAG HP; MINAMI K. (Ed.) Nutrição mineral em hortaliças. Campinas, SP: Fundação Cargill, p. 123-151.
GENÚNCIO GC; MAJEROWICZ N; ZONTA E; SANTOS AM; GRACIA D; AHMED CRM; SILVA MG. 2006. Crescimento e produtividade do tomateiro em cultivo hidropônico NFT em função da concentração iônica da solução nutritiva. Horticultura Brasileira 24: 175-179.

HOAGLAND DR; ARNON DI. 1950. The waterculture method for growing plants without soil. Berkeley, CA: Agric. Exp. Stn., Univ. of California. (Circ. 347).

HUETT DO. 1994. Growth, nutrient uptake and tipburn severity of hydroponic lettuce in response to electrical conductivity and $\mathrm{K}: \mathrm{Ca}$ ratio in solution. Aust. J. Agric. Res. 45: 251-267.

HUNT R. 1990. Basic growth analysis. London: Unwin Hyman. 112 p.

LUZ JMQ; GUIMARÃES STMR; KORNDÖRFER GH. 2006. Produção hidropônica de alface em solução nutritiva com e sem silício. Horticultura Brasileira 24: 295-300.
RESH HM. 2002. Hydroponic food production. 6 ed. Califórnia, EUA: Woodbridge Press. 567 p.

SANTOS OS. 1998. Soluções nutritivas. In: SANTOS SS. (Ed.). Hidroponia da alface. Santa Maria, RS: UFSM. p. 72-85.

SIDDIQI MV; KRONZUCKER HJ; BRITTO DT; GLASS DM. 1998. Growth of a tomato crop at reduced nutrient concentrations as a strategy to limit eutrophication. J. Plant Nutrition 21: 1879-1895.

SPOSITO G; MATTIGOD SV. 1980. GEOCHEM: a computer program for the calculation of chemical equilibria in soil solution and other natural water systems. Riverside, CA: Kearney Found. Soil Sci/Univ. of California. 